\title{
A Modification to the Copula Approach for Pricing Correlation-Dependent Credit Derivatives
}

\author{
Chih-wei Lee ${ }^{1}$ Cheng-kun Kuo ${ }^{2}$ \\ ${ }^{1}$ National Taipei College of Business \\ ${ }^{2}$ National Taiwan University
}

\begin{abstract}
This paper proposes a modified copula approach to defining correlation dependence used for pricing credit derivatives. For both single-name and multiname products, how default correlations react to common shocks should be appropriately measured. The copula approach is an effective method for this purpose.

However, in the currently available copula models, usually positive default correlations are allowed, or are relatively easy to implement. Consequently, these models may not provide sufficient information for pricing credit derivatives with mutually dependent defaults. In this paper, we explicitly define a default correlation structure that allows opposite response to a common factor. Using an exponential copula model as illustration, we show the modification is a more comprehensive description of default dependence found in market.
\end{abstract}

Keywords: copula, default correlation

\section{Introduction}

In pricing single-name credit instruments, it is necessary to consider the default dependence between the reference entity and the counterparty. In pricing multiname credit instruments, the effects of the default of an obligor on the remaining obligors must be addressed. For both single-name and multiname products, how default correlations react to common shocks should be appropriately measured.

For the three approaches proposed for estimating default correlation, the structural approach has the disadvantage of time-consuming numerical procedures, if asset default correlation is required. In implementing the reduced-form approach, the intensity function for each obligor has to be estimated.
However, there is usually not enough market data for the cross-section or time series analysis.

The copula approach specifies the joint distribution by simply taking the marginal distributions as given. It is increasingly popular in practice due to its simplicity in specifying the joint distribution. However, in the currently available copula models, usually positive default correlations are allowed, or are relatively easy to implement. Consequently, these models may not provide sufficient information for pricing credit derivatives with mutually dependent defaults

Under normal market conditions, firms usually exhibit similar responses to a common factor. But in other times, firms may show extremely different, or even opposite, responses to a common shock. This is evident when we witness a decrease in correlation. It is also documented that in a less competitive industry, the effect of a default may be beneficial due to less competition. Again, this leads to a decrease in correlation.

For example, the downgrades of Ford and General Motors to junk status by Standard \& Poor's in April 2005 result in a significant impact on default correlation. Average implied correlation in CDO equity tranches decreases from a level of around $24 \%$ in mid-April to around $12 \%$ on May 10 . Consequently, the market dynamics spelt trouble for hedge funds that followed a strategy based on inappropriate models. The losses on this strategy raise serious concern about the accuracy of current correlation models (See Patel (2005)).

In this paper, we explicitly define a default correlation structure that allows opposite response to a common factor. Using an exponential copula model as illustration, we show that the modification is a more comprehensive description of default dependence found in market. The modification not only can preserve the desired features of the copula approach, but also can be implemented with ease 
The remaining of the paper is organized as follows. Section 2 reviews and discusses the general merits of the current copula models used for estimating default correlation. Section 3 illustrates the deficiency found in these models using Giesecke (2003) model as an example. Section 4 concludes.

\section{Model Comparison}

The copula approach to construct a dependence structure is popular. In practice, it is usually combined with the structural and reduced-form approaches. When using a copula function to simulate correlated default times, survival functions have to be specified in a reduced-form or structural framework (See, for example, Turnbull (2005), and Jarrow and Deventer (2005)). The use of copula in credit risk literature was first made explicitly by Li (2000), followed by Mashal and Naldi (2001) and Schonbucher and Schubert (2001), among others. The Gaussian copula and the tcopula are the two frequently used copula functions. The Gaussian copula is the industry norm. Other copula functions proposed include: the factor copula, a special case of the Gaussian copula (Laurent and Gregory (2003)), the Archimedean copula (Rogge and Schonbucher (2003)), and the exponential copula (Giesecke (2003)).

When applying copula function to default correlation, we will refer to it as the survival copula. Denoting the joint survival probability $s\left(t_{1}, t_{2}\right)$ $=P\left(\tau_{1}>t_{1}, \tau_{2}>t_{2}\right)$, there exists a unique function $C^{\tau}$ : $[0,1]^{2} \rightarrow[0,1]$, called the survival copula of the default time vector $\left(\tau_{1}, \tau_{2}\right)$, such that the joint survival probability can be represented as $s\left(t_{1}, t_{2}\right)$ $=C^{\tau}\left(F_{1}\left(\tau_{1}\right), F_{2}\left(\tau_{2}\right)\right)$, where $F_{l}$ and $F_{2}$ represent the marginal distribution functions. The copula $C^{\text {r }}$ describes the default time dependence structure.

Using copula in dependence structure construction, like any model, has its limitations. For example, the original survival copula model and its extensions all have the disadvantage that there is no dynamics for credit spreads or their correlations. Also, there is no underlying economic rationale for the model. There is simple no physical interpretation for the copula correlation between two obligors.

When applying copula functions to pricing credit derivatives, it can result in relatively slow Monte Carlo simulations, especially for high ratings reference obligors. One-factor Gaussian copula is preferred among all copula functions because it allows for quick numerical integration techniques. Hull and White (2004) propose two alternative approaches: recurrence and bucketing, for implementing the model, in order to value an $n$-th to default credit default swaps or tranches of CDOs and indexes without Monte Carlo simulation.

\section{The Deficiency and the Modification}

In this section, we suggest a way to take into account possible negative correlation of firms reacting to a common factor.

\subsection{The deficiency}

There are various restrictive assumptions of same response to a common macro factor by different obligors as one common disadvantage of these models. In reality, firms may have different, or even opposite, responses to a common factor. In the following, we will use the Giesecke (2003) model to illustrate how to modify a model to take into account the opposite responses and remedy the deficiency found generally in the copula approach.

Giesecke uses bivariate exponential distribution commonly used in reliability theory to describe joint survival probability. The merits of his model are that it is given in closed-form and can be easily implemented. The efficient simulation of dependent default times for pricing and risk management purposes is straightforward as well.

Using Giesecke notations, suppose there are Poisson processes $N_{l}, N_{2}$, and $N$ with respective intensities $\lambda_{1}, \lambda_{2}$, and $\lambda$, where $\lambda_{i}$ is the idiosyncratic shock intensity of firm $i$, while $\lambda$ is the intensity of a macro-economic shock affecting both firms simultaneously. Then, default time $\tau_{i}$ of firm $i$ is

$$
\tau_{i}=\inf \left\{t \geq 0: N_{i}(t)+N(t)>0\right\}
$$

Using exponential copula, the joint survival probability can be obtained by

$$
\begin{aligned}
& \left.s\left(t_{1}, t_{2}\right)=H\left[\tau_{1}>t_{1}, \tau_{2}>t_{2}\right]=P N_{1}\left(t_{1}\right)=0, N_{2}\left(t_{2}\right)=0 N\left(t_{1} \vee t_{2}\right)=0\right] \\
& =\exp \left[-\lambda_{1} t_{1}-\lambda_{2} t_{2}-\lambda\left(t_{1} \vee t_{2}\right)=s_{1}\left(t_{1}\right) s_{2}\left(t_{2}\right) \min \left(e^{\lambda_{1}}, e^{\lambda_{2}}\right)\right]
\end{aligned}
$$

where $s_{i}(t)=P\left[\tau_{i}>t\right]=P\left[N_{i}(t)+N(t)=0\right]=e^{-\left(\lambda_{i}+\lambda\right) t}$. It can also be written as

$s\left(s_{1}^{-1}(u), s_{2}^{-1}(v)\right)=C(u, v)=\min \left(v u^{1-\theta_{1}}, u v^{1-\theta_{2}}\right)$

where $\theta_{i}=\frac{\lambda}{\lambda+\lambda}$.

As noted by Giesecke, in this model, defaults can only be positively related. This can be seen from equation (1) where default time correlation is 
governed by a common factor that can only have same impact on different obligors.

Negative default correlation of firms can be found in market encountering economic turbulence. Consequently, for modeling default correlation among a group of firms, it is natural to define various common factors and measures the responses of those firms to the common shocks. However, it is relatively difficult to incorporate common factors $N(t)$ in models using the Gaussian copula, the t-copula, and the Archimedean copula. Although the factor copula overcomes this problem, conditional independence has to be assumed. That is, $N_{i}(t)$ is assumed to be independent to each other conditional on the common factor. In the following, we modify the exponential copula approach of Giesecke (2003) to show how negative correlations can be incorporated.

\subsection{The modification} 2:

Consider default time $\tau_{l}$ and $\tau_{2}$ of firm 1 and firm

$$
\begin{aligned}
& \tau_{1}=\inf \left\{t \geq 0: N_{1}(t)+N(t)>0\right\} \\
& \tau_{2}=\inf \left\{t \geq 0: N_{2}(t)-N(t)>0\right\}
\end{aligned}
$$

That is, firm 2 responds differently to the common factor from firm 1 .

$$
\begin{aligned}
& P\left[N_{2}(k) \leq N(k)\right] \\
& =\sum_{i=0}^{\infty} \sum_{j=0}^{i} P\left[N(k)=i, N_{2}(k)=j\right] \\
& \approx e^{-\lambda k} \cdot e^{-\lambda_{2} k}+e^{-\lambda k}(\lambda k) \cdot\left(e^{-\lambda_{2} k}+e^{-\lambda_{2} k} \cdot \lambda_{2} k\right) \\
& =e^{-\left(\lambda+\lambda_{2}\right) k}+e^{-\left(\lambda+\lambda_{2}\right) k} \cdot \lambda k+e^{-\left(\lambda+\lambda_{2}\right) k}(\lambda k)\left(\lambda_{2} k\right)= \\
& e^{-\left(\lambda+\lambda_{2}\right) k}\left[1+\lambda k+(\lambda k)\left(\lambda_{2} k\right)\right]
\end{aligned}
$$

Thus we have

$$
\begin{aligned}
& s_{2}(t)=P\left[\tau_{2}>t\right]=\prod_{k=1}^{t} P\left[N_{2}(k) \leq N(k)\right] \\
& \cong \prod_{k=1}^{t} e^{-\left(\lambda+\lambda_{2}\right) k} \cdot\left(1+\lambda k+\lambda \lambda_{2} k^{2}\right) \\
& \cong e^{-\left(\lambda+\lambda_{2}\right) t(t+1) / 2}(1+\lambda t(t+1) / 2) \cong e^{-\lambda_{2} t(t+1) / 2}
\end{aligned}
$$

The approximation in equation (5) underestimates $P\left[N_{2}(k) \leq N(k)\right]$ by dropping some terms. It is noted that the resulting bias is acceptable for time horizon no more than five years and hazard rate less than $5 \%$. For example, for $\lambda_{2}=\lambda=5 \%$ and five years horizon, the approximate survival probability is $79.6 \%$, that under-estimates the true value $82.2 \%$ by $2.6 \%$. In fact, the maturity of most default- correlated products is shorter than five years, and the default probability, for firms with Moody's credit rating Baa or above, is under $5 \%$ over years 1970-2005, except for year 1986.

Therefore, for $t_{1}>t_{2}$, the joint survival probability is found to be

$$
\begin{aligned}
& s\left(t_{1}, t_{2}\right)=P\left[\tau_{1}>t_{1}, \tau_{2}>t_{2}\right]=P\left[N\left(t_{1}\right)=0, N_{1}\left(t_{1}\right)=0, N_{2}\left(t_{1}\right)=0\right] \\
& =\exp \left[-\left(\lambda+\lambda_{1}+\lambda_{2}\right) t_{1}\right]
\end{aligned}
$$

and for $t_{1}<t_{2}$,

$$
\begin{aligned}
& s\left(t_{1}, t_{2}\right)=P\left[\tau_{1}>t_{1}, \tau_{2}>t_{2}\right]=P\left[N\left(t_{1}\right)=0, N_{1}\left(t_{1}\right)=0\right] \\
& \prod_{k=t_{1}+1}^{t_{2}} P\left[N_{2}(k) \leq N(k)\right] \\
& \cong e^{-\left(\lambda+\lambda_{1}\right) t_{1}} \prod_{k=t_{1}+1}^{t_{2}} e^{-\left(\lambda+\lambda_{2}\right) k} \cdot\left(1+\lambda k+\lambda \lambda_{2} k^{2}\right) \\
& \cong e^{-\left(\lambda+\lambda_{1}\right) t_{1}} e^{-\left(\lambda+\lambda_{2}\right)\left(t_{2}+t_{1}+1\right)\left(t_{2}-1\right) / 2} e^{-\lambda\left(t_{2}+t_{1}+1\right)\left(t_{2}-1\right) / 2} \\
& =e^{-\left(\lambda+\lambda_{1}\right) t_{1}} e^{-\lambda_{2}\left(t_{2}^{2}-t_{1}^{2}+t_{2}-t_{1}\right) / 2}
\end{aligned}
$$

These survival probabilities can then be used to compute the linear correlation of the default indicator variables:

$$
\rho\left(1_{\left\{\tau_{1} \leq t\right.}, 1_{\left\{\zeta_{2} \unlhd\right\}}\right)=\frac{s(t, t)-S_{1}(t) S_{2}(t)}{\sqrt{\left(1-S_{1}(t)\right) S_{1}(t)\left(1-S_{2}(t)\right) S_{2}(t)}}
$$

Following the discussion of Giesecke, default indicator correlation can lead to severe misinterpretations of the true default correlation structure, as pointed by Embrechts, McNeil and Straumann (2001). Defined on the level of the copula, Spearman's rank correlation $\rho^{S}$ does not have the same problems.

$$
\text { Denoting } s_{1}\left(t_{1}\right)=u, s_{2}\left(t_{2}\right)=v \text {, and }
$$

$\theta=\lambda_{2} /\left(\lambda+\lambda_{1}\right)$, we obtain $t_{1}=-\ln u /\left(\lambda+\lambda_{1}\right)$, $t_{2}=\left(\sqrt{1-\frac{4 \ln v}{\lambda_{2}}}-1\right) / 2$. Thus, $s\left(t_{1}, t_{2}\right)=u^{1+\theta}$ when $t_{1}>t_{2}$, and $s\left(t_{1}, t_{2}\right)=u^{1-\theta} v$ when $t_{1}<t_{2}$. Besides, if $t_{1}>t_{2}$ and $t_{2} \leq 3$, we have

$$
\begin{aligned}
& \frac{u^{1+\theta}}{u^{1-\theta} v}=\frac{u^{2 \theta}}{v}=\frac{\exp (2 \theta \ln u)}{\exp (\ln w)} \\
& =\frac{\exp \left(-2 \lambda_{2} t_{1}\right)}{\exp \left(-\lambda_{2}\left(t_{2}^{2}+t_{2}\right) / 2\right)} \leq \frac{\exp \left(-2 \lambda_{2} t_{1}\right)}{\exp \left(-2 \lambda_{2} t_{2}\right)}<1
\end{aligned}
$$


Therefore, the survival copula becomes $C^{\tau}(u, v)$ $=s\left(s_{1}^{-1}(u), s_{2}^{-1}(v)\right) \cong \min \left(u^{1+\theta}, u^{1-\theta} v\right)$. Denoting the equivalent copula by $K^{\tau}$, then

$K^{\tau}(u, v)=C^{\tau}(1-u, 1-v)+u-v+1$

$=\min \left[(-u)^{1+\theta},(1-u)^{1-\theta}(1-v)\right]+u+v-1$

Therefore, Spearman's rank correlation $\rho^{S}$, for two firms with opposite response to the joint shock, is simply the linear correlation of the copula $K^{\tau}$, given by:

$$
\begin{aligned}
& \rho^{S}\left(\tau_{1}, \tau_{2}\right)=\rho\left(p_{1}\left(\tau_{1}\right), p_{2}\left(\tau_{2}\right)\right)= \\
& 12 \int_{0}^{1} \int_{0}^{1} K^{\tau}(u, v) d u d v-3 \\
& =12\left[\frac{1}{(2+\theta)\left(\frac{3}{2}+\frac{1}{\theta}\right)}-\frac{1}{(2-\theta)\left(\frac{3}{2}+\frac{1}{\theta}\right)}+\frac{1}{2(2-\theta)}\right]^{-3} \\
& =\frac{9 \theta^{2}-24 \theta+12-\frac{20}{\theta}}{(2+\theta)(2-\theta)\left(3+\frac{2}{\theta}\right)}
\end{aligned}
$$

where $p_{i}(t)=P\left[\tau_{i} \leq t\right]=1-s_{i}(t)$ is the distribution function of $\tau_{i}$.

In Figure 1, rank default correlation of two firms with opposite response to the joint shock and that with same response are compared. Rank default correlation is plotted as functions of the joint shock intensity $\lambda$. We set $\lambda_{1}=0.01, \lambda_{2}=0.02$, which corresponds to a one-year default probability of about $1 \%$ and $2 \%$ when $\lambda=0$. With increasing $\lambda$, the default correlation increases for both firms with positive and opposite common shock response, due to the common shock component of the default risk dominating the idiosyncratic component. The result for the firm with positive common shock response is consistent with Giesecke's. Therefore, the modification is a more comprehensive description of default dependence found in market.

\section{Figure 1}

Comparison of Rank Default Correlation for Firms with Same Response and Opposite Response to the Common Shock

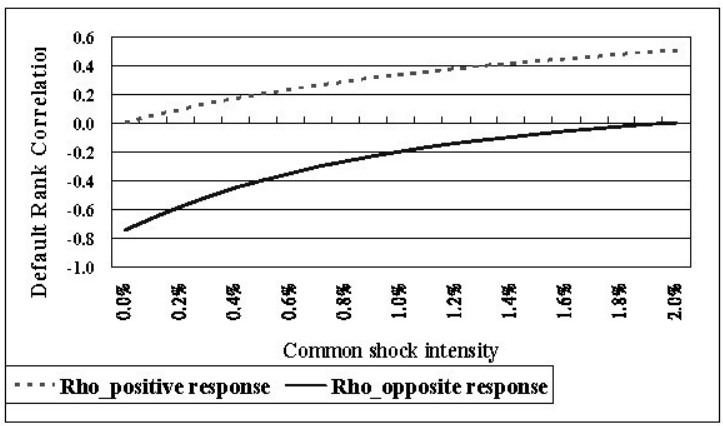

\section{Conclusion}

Without involving in the complicated problem of calibration and parameter estimation in the implementation of the structural and reduced-form approach, the copula approach becomes the market's standard for valuing correlation dependent credit derivatives. However, the copula models are often restricted to positive correlation specification. The modification we propose in this paper is one suggestion to handle default correlation structure in which there are opposite responses to a common macro factor. Other copula models may need different approach to evaluate the effect of common shocks so as to model negative default correlations.

\section{References}

[1] Duffie, D. and N. Garleanu. "Risk and Valuation of Collateralized Debt Obligations." Financial Analysts Journal, 57, 1 (2001), pp. 41-59.

[2] Embrechts, P., A. McNeil and D. Straumann. "Correlation and Dependency in Risk Management, in M. Dempster, ed., Risk Management: Value at Risk and beyond, Cambridge University Press, 2001. Giesecke, K. "A Simple Exponential Model for Dependent Defaults." The Journal of Fixed Income, 13, 3 (2003), pp. 74-83.

[3] Hull , J. and A. White. "Valuation of a CDO and an N- th to Default CDS without Monte Carlo Simulation." Journal of Derivatives, 12, 2 (2004), pp. 8-23.

[4] Jarrow, R. and D. van Deventer. "Estimating Default Correlations using a Reduced-Form Model." Risk, 18, 1 (2005), pp. 83-87.

[5] Laurent J.-P. and J. Gregory. "Basket Default Swaps, CDOs, and Factor Copulas." Working paper, University of Lyon, 2003.

[6] Li, D. "On Default Correlation: a Copula Function Approach." The Journal of Fixed Income 9, 3 (2000), pp. 43-54.

[7] Mashal, R., and M. Naldi. "Pricing Multiname Credit Derivatives: Heavy Tailed Hybrid Approach." Working paper Columbia Business School, 2001. Patel, N. "Crisis of Correlation." Risk 18, 6 (2005), pp.46-48.

[8] R.ogge, E. and P. J. Schonbucher "Modeling Dynamic Portfolio Credit Risk." Working paper, ABN AMRO Bank and ETH Zurich, 2003

[9] Schonbucher, P., and D. Schubert. "Copula-Dependant Default Risk in Intensity Models." Working paper, Bonn University, 2001.

[10] Turnbull, S. M. "Unresolved Issues in ModelingCredit-Risky Assets." The Journal of Fixed Income, 15, 1 (2005), pp. 68-87. 